\title{
Desafios e benefícios do uso da aprendizagem baseada em problemas na educação a distância: uma revisão integrativa de literatura
}

\author{
Challenges and benefits of using problem-based learning in distance education: an integrative
} literature review

Desafíos y beneficios del uso del aprendizaje basado en problemas en la educación a distancia: una revisión integrativa de la literatura

Juan Magalhães Paiva

ORCID: https://orcid.org/0000-0002-5775-1548 Universidade Federal do Maranhão, Brasil

E-mail: juanunasus@gmail.com

Sannya Fernanda Nunes Rodrigues

ORCID: https://orcid.org/0000-0002-6492-7732 Universidade Estadual do Maranhão, Brasi

E-mail: rodriguessannya@gmail.com

João Batista Bottentuit Junior

ORCID: https://orcid.org/0000-0002-4432-0271

Universidade Federal do Maranhão, Brasil E-mail: jbbj@terra.com.br

Daniel Formiga

ORCID: https://orcid.org/0000-0002-0220-7211

Universidade Federal do Maranhão, Brasil

E-mail: daniel.formiga@discente.ufma.br

Karla Cristina dos Santos Ferreira Ataide Lima

ORCID: https://orcid.org/0000-0002-0382-2910

Universidade Federal do Maranhão, Brasil E-mail: ferreira.karla@ufma.br

Carlos Guilherme Moraes Cerqueira

ORCID: https://orcid.org/0000-0001-7873-4539

Universidade Federal do Maranhão, Brasil

E-mail: carlos.cerqueira@discente.ufma.br

Karla Patrícia Bernardes Ferreira Lima

ORCID: https://orcid.org/0000-0002-3438-4909

Universidade Estadual do Maranhão, Brasil

E-mail: karlapbflima@gmail.com

Juliane Silva Santos

ORCID: https://orcid.org/0000-0002-8849-8522

Universidade Federal do Maranhão, Brasil E-mail: julianejolie7@gmail.com

\begin{abstract}
Resumo
As alterações proporcionadas pelo avanço tecnológico levaram à necessidade de desenvolvimento de novas formas de transmissão de informações e ensino, com o grande destaque para a Educação à Distância (EaD). A isso se somam inovadoras metodologias de ensino que buscam romper o padrão tradicional de educação que tem papéis fixos de professor e aluno, tais como a Aprendizagem Baseada em Projetos (ABP), recurso que propõe uma nova forma de ensinar através da solução de quesitos práticos pelos alunos. Dessa forma, o presente artigo objetivou responder à seguinte pergunta norteadora: "como a Aprendizagem Baseada em Problemas vêm sendo aplicada no âmbito da educação a distância e quais são os benefícios e desafios de seu uso?". Para isso, foi efetuada revisão integrativa de literatura acadêmica relacionada as experiências com a ABP em diferentes áreas e graus de escolaridade. Ao final, foi possível identificar que o uso desta ferramenta metodológica de ensino na EaD gerou benefícios e manifestos no contexto de sua aplicabilidade, tais como: melhor gestão de tempo; desempenho do discente; aulas mais dinâmicas; aumento da participação e atenção dos alunos; análise mais críticas em solucionar e apresentar hipóteses para as atividades propostas; melhor compreensão do conteúdo; maior flexibilidade no eixo colaborativo de ensino. Por outro lado, também se reconheceram dificuldades, a exemplo de: limitações de recursos; carência de domínio técnico, habilidades e manuseio de ferramentas tecnológicas por parte dos participantes e resistência do uso da metodologia.
\end{abstract} Palavras-chave: Aprendizagem Baseada em Problemas; Educação a Distância; Ensino; Metodologia de Ensino. 


\begin{abstract}
The changes brought about by technological advances led to the need to develop new ways of transmitting information and teaching, with great emphasis on Distance Education. Added to this are innovative teaching methodologies that seek to break the traditional pattern of education that has fixed roles of teacher and student, such as Project-Based Learning (PBL), resource that proposes a new way of teaching through the solution of practical questions by students. Thus, this article aimed to answer the following guiding question: "How has Problem-Based Learning been applied in the context of distance education and what are the benefits and challenges of its use?". For this, an integrative review of academic literature related to experiences with PBL in different areas and levels of education was carried out. In the end, it was possible to identify that the use of this methodological tool for teaching on Distance Education generated benefits and manifests in the context of its applicability, such as: better time management; student performance; more dynamic classes; increased student participation and attention; more critical analysis in solving and presenting hypotheses for the proposed activities; better understanding of the content; greater flexibility in the collaborative teaching axis. On the other hand, difficulties were also recognized, such as: resource limitations; lack of technical domain, skills and handling of technological tools by the participants and resistance to the use of the methodology.
\end{abstract}

Keywords: Project-Based Learning; Distance Education; Teaching; Teaching Methodology.

\title{
Resumen
}

Los cambios provocados por los avances tecnológicos llevaron a la necesidad de desarrollar nuevas formas de transmitir información y enseñar, con gran énfasis en la Educación a Distancia (ED). A esto se suman metodologías de enseñanza innovadoras que buscan romper el patrón tradicional de educación que tiene roles fijos de docente y alumno, como el Aprendizaje Basado en Proyectos (PBL), un recurso que propone una nueva forma de enseñar a través de la solución de requerimientos prácticos por los estudiantes. Así, este artículo tuvo como objetivo responder a la siguiente pregunta orientadora: “¿cómo se ha aplicado el Aprendizaje Basado en Problemas en el ámbito de la educación a distancia y cuáles son los beneficios y desafíos de su uso?”. Para ello, se realizó una revisión integradora de literatura académica relacionada con experiencias con ABP en diferentes áreas y niveles educativos. Al final, se pudo identificar que el uso de esta herramienta metodológica de enseñanza en la educación a distancia generó beneficios y se manifiesta en el contexto de su aplicabilidad, tales como: mejor manejo del tiempo; desempeño de los estudiantes; clases más dinámicas; mayor participación y atención de los estudiantes; análisis más crítico en la resolución y presentación de hipótesis para las actividades propuestas; mejor comprensión del contenido; mayor flexibilidad en el eje de la enseñanza colaborativa. Por otro lado, también se reconocieron dificultades como: limitaciones de recursos; falta de dominio técnico, destreza y manejo de herramientas tecnológicas por parte de los participantes y resistencia al uso de la metodología.

Palabras clave: Aprendizaje Basado en Problemas; Educación a distancia; Enseñanza; Metodología de la Enseñanza.

\section{Introdução}

As recentes mudanças que ocorrem na sociedade despertam consequências significativas que estão modificando o comportamento e atitudes do homem contemporâneo, principalmente na relação de ensino e aprendizagem. As novas tecnologias estão assumindo papéis importantes e cada vez mais exigindo novas posturas e atuações para essa nova realidade em diversos campos sociais. Percebe-se que, com a ascensão da tecnologia, a informação ganha destaque e relevância para os parâmetros de desenvolvimento, “[...] onde o conhecimento é um recurso flexível, fluido, sempre em expansão e mudança” (Hargreaves, 2003, p. 37).

Atualmente, vivenciamos uma grande revolução tecnológica marcada por uma "sociedade de informação", "sociedade informatizada", "sociedade do conhecimento", ou "sociedade do saber" (Gentili, 1996, p. 31). O presente e o futuro se entrelaçam numa reinvenção constante para atender novos modelos, padrões, processos e cenários. Em paralelo as essas transformações, a prática de ensinar tem criado diversos procedimentos, gerando inquietações e alterações nos pontos de vista de docentes e instituições de ensino.

Essa transfiguração digital e tecnologizada são um dos grandes obstáculos enfrentados em todos os setores sociais, seja nos ambientes empresariais corporativos ou públicos (Seufert; Meier, 2016). A tendência da educação é passar por uma reconfiguração na maneira de ensinar que está intrinsicamente ligada com vários aspectos e paradigmas. Conforme Chigona 
(2018), os procedimentos de ensino e aprendizagem demandam novos saberes e metodologias que não são possíveis de serem aplicados nos parâmetros tradicionais de ensino, diante dessa transformação digital.

Quando pensa-se na prática de aprender, Meirieu (1999, p. 79) destaca que é “[...] uma interação que nunca é uma simples circulação de informações, um sujeito e o mundo, um aprendiz que já sabe sempre alguma coisa e um saber que só existe porque é reconstruído". A aprendizagem também passa por mudanças necessárias para poder se adaptar em uma sociedade que está em constante mudança social e tecnológica. Andrade e Sousa (2016) já afirmavam que diante das demandas vigentes, os processos de ensino e aprendizagem tradicionais não são mais aplicados, devido às mudanças de perfis e projeções dos alunos do século XXI.

É notório que a dinâmica educacional vem trazendo novos aprendentes através de métodos e instrumentos dentro de um eixo pedagógico que estabelece e redefine padrões de ensino. Desta forma, a Educação a Distância (EaD) tem demonstrado diversas abordagens para atender a uma demanda emergente que está em busca constante por informação e necessária autonomia nas diversificadas instâncias educacionais.

O processo educacional à distância, conforme Mugnol (2009, p. 03), “[...] é reconhecido como centrado no aluno e mediado pelas tecnologias da sociedade da informação”. Moore e Kearsley (2007) destacam que a EaD é um elo constituído de um diálogo autônomo por intermédio de dispor de técnicas e instrumentos necessários de comunicação para mediar um conhecimento à distância. Oliveira $(2007$, p. 69) define que a EaD trata-se:

[...] de uma estratégia educativa alicerçada na utilização de novas tecnologias, no estímulo às estruturas cognitivas operatórias flexíveis e em métodos pedagógicos que permitem que as condições inerentes ao tempo, espaço, ocupação e idade dos estudantes não sejam condicionantes ou impeditivos para a aprendizagem.

A EaD reconfigura novas formas e práticas de ensinar, o que possibilita seu exercício mediado pelas tecnologias digitais, dentro de uma estrutura pedagógica e didática. No entanto, um dos desafios da educação é a utilização de propostas pedagógicas e de metodologias que estimulem o processo de ensino e aprendizagem, analisando a exaustão do modelo tradicional de ensino (Santos et al, 2016). Hoje, buscam-se alternativas estratégicas e modelos pedagógicos para trabalhar o perfil de alunos que estimulem a prática cognitiva, a compreensão do conhecimento transmitido e a participação efetiva dentro do contexto de ensino na EaD.

Conforme Mugnol (2009, p. 10), “[...] a educação a distância se desenvolve através da articulação de atividades pedagógicas capazes de desenvolver os aspectos afetivo, psicomotor e cognitivo dos estudantes". O emprego de metodologias na $\mathrm{EaD}$ coloca o aluno num papel consciente, decorrente das atividades acadêmicas na direção de seu aprendizado. Moran (2015, p. 17) destaca: para que a proatividade dos alunos seja legítima, precisa-se “[...] adotar metodologias em que os alunos se envolvam em atividades cada vez mais complexas e que tenham que tomar decisões e avaliar os resultados com apoio de materiais relevantes."

Sustentando a ideia levantada por Moran, determinadas metodologias podem ser compreendidas como ativas, pois orientam os processos de ensino e aprendizagem que se concretizam em estratégias, abordagens e técnicas concretas, específicas e diferenciadas (Moran; Bacich, 2018). Essas metodologias consistem numa possibilidade que motiva a independência do aluno, oposta ao modelo tradicional de ensino, diante das habilidades e concepções centradas na aprendizagem significativa (Paiva et al, 2016).

Um tipo de metodologia ativa que tem sido abordado amplamente no ensino à distância e que corresponde ao cenário inovador é a chamada Aprendizagem Baseada em Problemas (ABP). "Esse método contém forte motivação prática e estímulo cognitivo para gerar soluções criativas e pode ser aplicado tanto na forma de ensino tradicional, quanto no ensino a distância" (Mezzari, 2011, p. 115). 
A aprendizagem baseada em problemas é idealmente apropriada para aprendizagens centradas no estudante, autodirigidas e individualizadas. Em um modelo centrado no estudante, os discentes podem escolher um problema específico ou tema maior. Eles, então, projetam, desenvolvem e modificam o modo ou caminho da resolução do problema. Isto inclui decisões sobre o que deve ser aprendido, quais recursos devem ser procurados e usados e como a comunicação do entendimento e resolução do problema deve ser apresentada. Os professores atuam como facilitadores e colaboradores (Glasgow, 2019, p. 35-36).

Assim, compreende-se que a metodologia da ABP tende a beneficiar o conhecimento prévio dos discentes, estimulando uma área indagativa da aprendizagem por meio da elaboração de evidências, experiências e hipóteses.

Este artigo se concentra numa revisão integrativa de literatura que consiste analisar a contribuição da aplicabilidade deste instrumento metodológico colaborativo, através de seu uso em diferentes cenários da EaD. Dessa forma, levantaram-se as seguintes questões norteadoras: "Como a Aprendizagem Baseada em Problemas vêm sendo aplicada no âmbito da educação a distância? Quais são os benefícios e desafios de seu uso?". Partindo desse pressuposto da pesquisa, pretende-se apresentar discussões dos resultados e objetivos do processo integrativo da ABP na EaD, assim como, destacar seus benefícios e desafios.

\section{Metodologia}

O presente estudo versa numa revisão integrativa de literatura de fontes secundárias, por meio de levantamento bibliográfico relativo às experiências realizadas ao objeto pesquisado. De acordo com autores Alcoforado et al. (2014, p. 9), “[...] a revisão integrativa de literatura é um método que tem como finalidade sintetizar resultados obtidos em pesquisas sobre um tema ou questão, de maneira sistemática, ordenada e abrangente”.

Essa metodologia de pesquisa permite uma associação relativa à integração de efeitos de estudos significativos realizados na prática (Souza et al, 2010). Botelho, Cunha e Macedo (2011) apresentam 6 etapas que consistem na revisão integrativa de literatura, podendo destacar:

1. Identificação do tema e seleção da questão de pesquisa;

2. Estabelecimento de critérios de inclusão e exclusão;

3. Identificação dos estudos pré-selecionados e selecionados;

4. Categorização dos estudos selecionados;

5. Análise e interpretação dos resultados;

6. Apresentação da revisão/ síntese do conhecimento.

Para tanto, foi considerada a seguinte pergunta norteadora: “como a Aprendizagem Baseada em Problemas vêm sendo aplicada no âmbito da educação a distância e quais são os benefícios e desafios de seu uso?”.

A busca de trabalhos publicados consistiu em consulta às bases de periódicos e repositórios, tais como: Resdite (Revista de Saúde Digital e Tecnologias Educacionais), RIU (Repositório Institucional da Unipampa), Revista Multitexto (Centro de Educação a Distância da Universidade Estadual e Montes Claros), SciELO (Scientific Electronic Library Online), SCOPUS Databases e LOCUS (Repositório Institucional da Universidade Federal de Viçosa).

Nas buscas, foram adotados os descritores: Educação a Distância; Aprendizagem Baseada em Problema; problem based learning; distance education; EaD; APB; PBL.

O levantamento nas bases de dados foi realizado entre maio e julho de 2021, utilizando o conectivo "and”. Tal termo permite localizar publicações com combinação mais restritiva, diante dos descritores pesquisados. Seguindo os critérios de inclusão e exclusão, foram excluídas as publicações que não apresentaram diretamente resultados correlacionados ao objeto deste estudo e trabalhos repetidos. Para os critérios de inclusão usou-se:

a) Recorte temporal nos últimos 10 anos (2011 a 2021);

b) Texto integral disponível em formato eletrônico, gratuito e redigido em português e/ou inglês; 
c) Ser compatível com no mínimo um dos objetivos da pesquisa, isso é, contemplar os cenários dos resultados quanto aos benefícios e desafios da aplicabilidade da metodologia da Aprendizagem Baseado em Problema na Educação a Distância.

\section{Resultados e Discussão}

Diante das buscas realizadas nos principais periódicos e repositórios apresentados, foram encontrados 22 trabalhos de pesquisa, incluindo: Artigos, Monografias, Dissertações e Teses. Após análise crítica dos trabalhos compreendidos nesta revisão integrativa, buscou-se delimitar os resultados para que a interpretação fosse compatível com as variáveis construídas, tais como: autor(a)(es); ano; tipo de trabalho e/ou pesquisa; objetivo; resultado; benefícios e desafios, obtendo, desta forma, a amostra final de 9 trabalhos selecionados, conforme evidencia-se no Quadro 1 abaixo:

Quadro 1: Buscas de Trabalhos

\begin{tabular}{|l|c|c|}
\hline \multicolumn{1}{|c|}{ PERIÓDICO/REPOSITÓRIO } & BUSCAS & SELECIONADOS \\
\hline Resdite (Revista de Saúde Digital e Tecnologias Educacionais & 3 & 1 \\
\hline RIU (Repositório Institucional da Unipampa) & 2 & 1 \\
\hline SCOPUS Databases & 5 & 3 \\
\hline $\begin{array}{l}\text { Revista Multitexto (Centro de Educação a Distância da Universidade } \\
\text { Estadual e Montes Claros) }\end{array}$ & 3 & 1 \\
\hline SciELO (Scientific Electronic Library Online) & 7 & 2 \\
\hline $\begin{array}{l}\text { LOCUS (Repositório Institucional da Universidade Federal de } \\
\text { Viçosa) }\end{array}$ & 2 & 1 \\
\hline \multicolumn{1}{|c|}{ TOTAL } & $\mathbf{2 2}$ & $\mathbf{9}$ \\
\hline
\end{tabular}

Fonte: Elaborado pelos autores (2021).

Com o resultado da amostra final dos 9 trabalhos identificados, 7 estavam no formato de artigo científico, 1 como trabalho de conclusão de curso de graduação (monografia) e 1 no formato de Tese (doutorado), sendo seis no idioma português, e três em inglês. Para melhor organização desta pesquisa, procurou-se apresentar nos trabalhos selecionados evidências quanto às suas origens, destacando desta forma: autor; título do trabalho; ano; tipo de trabalho e seu principal objetivo, conforme estão descritos mais detalhadamente na Tabela 1 abaixo: 
Tabela 1: Identificação dos Trabalhos Selecionados
ORDEM
AUTOR(ES)
TÍTULO DO TRABALHO
TIPO DE
ANO TRABALHO
OBJETIVO

1 BOZIC, N.; WILLIAMS, Online problem based and enquiry based learning in the H. training of educational psychologists

O uso da Aprendizagem Baseada em Problemas (ABP) como reforço ao ensino presencial utilizando o ambiente de aprendizagem Moodle

Educação à distância em nefrologia na Amazônia: processos e resultados

A comparison of classroom and online asynchronous problem-based learning for students undertaking statistics training as part of a Public Health Masters degree

TAKATA, Y.; et al.

Content analysis of medical students' seminars: a unique method of analyzing clinical thinking

Aprendizagem baseada em problemas/projetos em ambiente online na perspectiva de educadores e educandos da Ciência dos Alimentos

Educação Popular e Aprendizagem Baseada em

ALMEIDA, C. R. S.; CAMARGO, M. N.; CAMARGO, L. B.

PORTELA, N. M.; COSTA, J. M. B. S.; MAGALHÃES, G. S. G.

RICHARDT, C. C. L. T.
Problemas na EaD: uma aplicação no curso de ciências sociais EaD/UNIMONTES

A experiência com o uso do e-learning na aprendizagem baseada em problemas de um curso de medicina

Aprendizagem Baseada Em Problemas (ABP): proposta de aplicação prática para o ensino de português na educação a distância
$2011 \quad$ Artigo

Artigo

Relatar como a ABP tem sido empregada de forma online na formação do Psicólogo na Universidade de Birmingham, Reino Unido.

Aplicar e discorrer o uso da ferramenta metodológica da ABP em um Ambiente Virtual de Aprendizagem de uma disciplina de Parasitologia e Micologia Médica do curso de Medicina da Universidade Federal de Ciências da Saúde de Porto Alegre (UFCSPA).

Relatar experiências dos alunos no curso semipresencial de nefrologia para estudantes de graduação em Medicina que fez uso em sua metodologia da Aprendizagem Baseada em Projetos (ABP).

Comparar o uso da ABP no ensino presencial tradicional versus aprendizagem online assíncrona em um grupo de estudantes em nível de mestrado em Saúde Pública da Universidade de Maastricht na Holanda.

Analisar as habilidades comunicacionais de estudantes de medicina asiáticos durante seminários em webinário estruturados sob a metodologia da Aprendizagem Baseada em Problemas (ABP), com o propósito de examinar o desenvolvimento de seu pensamento crítico.

Analisar a aplicabilidade da ABP em um projeto educacional de

2013 Tese Ciência dos Alimentos, abrangendo docentes e discentes de um curso técnico

Analisar a viabilidade da aplicação da ABP em disciplinas ofertadas em um curso de Licenciatura em Ciências Sociais, evidenciando suas vantagens e desvantagens metodológicas na perspectiva dos docentes e discentes.

Relatar a experiência do uso de recursos de e-learning na ABP em um curso de medicina

Apresentar proposta de aplicação da ABP para o ensino de português na educação a distância (EaD), sob a ótica de Dewey e Irandé Antunes

Fonte: Elaborada pelos autores (2021). 
Entre os trabalhos selecionados, observou-se o objetivo de cada publicação: seis artigos trataram da ABP como uma metodologia aplicada e um componente educacional que auxiliou o estudante em sua formação na área das Ciências Médicas, podendo observar a graduação em psicologia, medicina e a nível de pós-graduação (mestrado) em Saúde Pública. Apenas um artigo tratava dessa experiência voltada para um outro eixo educacional que abrange a área das Ciências Humanas, no campo das ofertas de disciplinas em um curso de Graduação em Licenciatura em Ciências Sociais. A única tese selecionada apresentou a aplicabilidade da ABP em um curso técnico de ensino e o TCC destacou uma proposta de ensino da língua portuguesa para o ensino médio escolar.

Através dos trabalhos analisados, constatou-se a carência do uso desta metodologia ativa de ensino em outros eixos de ensino e formação na Educação a Distância, mas se evidencia que a abordagem pode ter uma perspectiva heterogênea, evidenciando a versatilidade desta ferramenta. Em todos os trabalhos que usavam ambientes virtuais de aprendizagem, foram apresentados objetos de aprendizagem como: vídeos, textos, fóruns, questionários, chats, animações e mediação pelo tutorprofessor online ou presencial, que tirava as dúvidas relacionadas com as propostas lançadas através da Aprendizagem Baseada em Problemas. Todos os participantes progrediam, conforme o prazo determinado e a execução crítica de pensamento e análise.

Com a identificação da $\mathrm{ABP}$ nas questões relacionadas no campo da $\mathrm{EaD}$, pode-se observar que, para alcançar os objetivos propostos, foram atribuídas práticas metodológicas de forma sistemática, que pontuam os benefícios e desafios compreendidos no uso deste instrumento. Seguindo o que fora problematizado neste artigo de revisão integrativa de literatura, os trabalhos analisados apontaram interessantes situações que foram compiladas na Tabela 2:

Tabela 2: Benefícios e Desafios do uso da ABP na EaD.

\section{ORDEM}

- Tempo para reflexão do problema proposto (opinião) para ser discutido;

1 - A flexibilidade do método online é atraente;

- Interatividade da discussão;

- Maior frequência das mensagens.

- Possibilidade do aluno estudar o conteúdo proposto em qualquer lugar e/ou hora;

2

- Adaptação do Ritmo do aluno;

- Contribuição e realização de esclarecimentos de dúvidas, diante das atividades propostas, sem ônus para o docente e discente.

- Maior dinamicidade e interação entre os discentes;

- Aumento nas discussões nos fóruns disponibilizados;

- Acessibilidade dos docentes no decorrer do curso;

- Maior qualidade dos recursos didáticos;

- Apresentação de casos clínicos (problemas);

- Dinâmica nas discussões;

- Contribuições em ministrar uma determinada disciplina, onde apresenta uma certa carência na forma presencial.
- Capacidade de aplicar pesquisa para a prática ou abordagem de problemas mal definidos

- Carência de inclusão desta metodologia na formação complementar em outras áreas de forma online.

- Necessidade do docente em estar acompanhando o processo de aprendizagem, diante das habilidades e competências adquiridos pelos discentes;

- Maior qualificação do docente em apresentar informações completas para o desenvolvimento para a resolutividades da atividade proposta;

- Carência no uso desta metodologia como parte complementar de ensino e aprendizagem.

- Apresentação de problemas com instrumentos e/ou equipamentos de acesso às problematizações propostas online. 


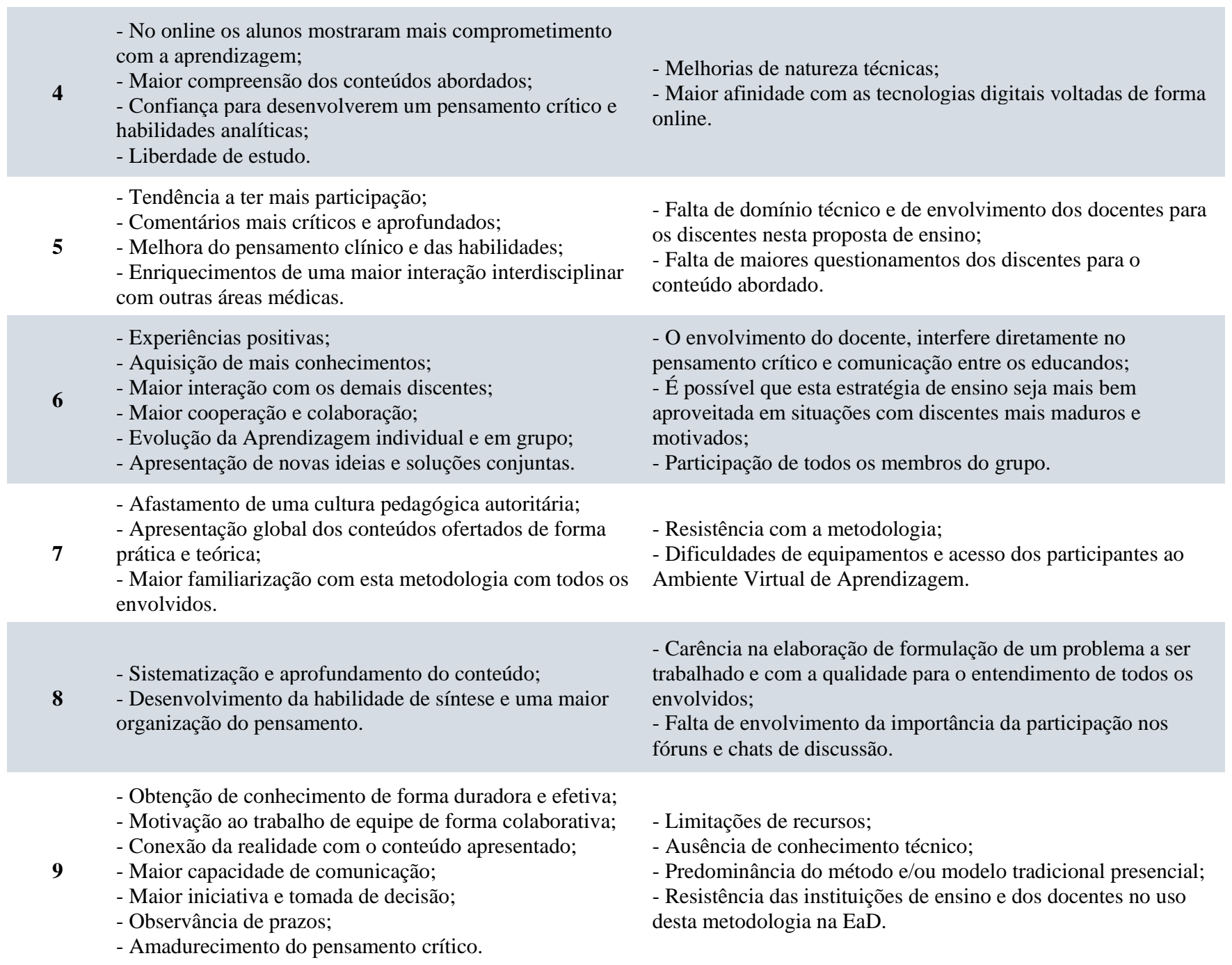

Fonte: Elaborada pelos autores (2021).

\section{Discussão}

A contextualização das origens da Aprendizagem Baseada em Problemas como metodologia de ensino é de fundamental importância para que seu entendimento e compreensão sejam discutidos e abordados nas práticas encontradas na Educação a Distância em diferentes cenários de uso contemporâneo. A ABP deriva do termo anglófono Problem-Based Learning (PBL) e a sua evolução está intrinsicamente ligada às práticas de ensino na área médica.

A fase embrionária da ABP ocorreu em meados da década de 60 na University Faculty of Health Sciences, localizada no Canadá, onde, em um curso de medicina, foi adotada nova estratégia, formando um comitê de alunos que tinham anseio de evidenciar novas prática nas estruturas e formas curriculares de ensino e aprendizagem que lhes eram transmitidos. Segundo Dias (2016, p. 54 e 55), esse comitê “[...] tinha como objetivo permitir que seus estudantes de medicina tivessem habilidades para resolver problema, conhecer, avaliar, juntar, interpretar e aplicar uma grande quantidade de informações que trouxessem respostas mais concisas e rápidas aos pacientes".

Apesar de um recorte temporal neste artigo de 10 anos, diante das publicações vinculadas e da importância literária voltada para a prática do emprego da $\mathrm{ABP}$ na $\mathrm{EaD}$, constatou-se que a formação auxiliada com as técnicas desta metodologia vem sendo cada vez mais utilizada nas áreas de ensino médico. Com o tempo, a partir das experiências dos cursos de medicina 
canadenses, a ABP passou a ser introduzida em diferentes instituições de ensino, para muitas outras escolas de medicina e para diversas áreas do conhecimento.

Para Gil (2009), esta prática metodológica deu um impulso ao suporte didático e atuação de discentes em contextos diferentes de realidades, diante das atividades "problemas" apresentados em sala de aula. Tortella e Borochovicius (2014, p. 268) complementam que "[...] a ABP tem como premissa básica o uso de problemas da vida real para estimular o desenvolvimento conceitual, procedimental e atitudinal do discente".

De maneira oposta ao ensino tradicional, a ABP mantém o aluno na criação do conhecimento em circunstâncias recorrentes e perceptíveis, pois "[...] é mais vantajoso ensinar o educando a aprender do que arriscar transmitir-lhe todos os conceitos e esperar que ele os incorpore à prática no futuro" (Ribeiro, 2005, p. 30).

Com a expansão e popularização desse método, onde se coloca o educando no centro deste processo de ensino, Jong et al (2013) apresentam quatro elementos fundamentais a serem utilizados na ABP, para que o entendimento seja mais bem repassado: o método deve ser contextualizado, colaborativo, autodirigido e construtivo.

Hoje, por meio da aprendizagem online, é possível traçar muitos parâmetros metodológicos para viabilizar a aprendizagem, diante dos recursos possíveis de serem executados nos diversos níveis de ensino. A flexibilidade dessa aprendizagem tem a faculdade de romper os limites físicos proeminentes ao modelo do ensino presencial. Também sugere-se que, quando alinhadas às metodologias de ensino com estratégias de ensino online ou à distância, os alunos também desenvolvem competências técnicas importantes, como pesquisa na internet, alfabetização em informática e habilidades de autoaprendizagem, além de um senso aprimorado de autoeficácia como resultado de sua experiência online da aprendizagem (Jong et al, 2013).

As decisões sobre a estrutura e o foco dos módulos ou cursos online devem, portanto, ser baseadas no tipo de aprendizagem desejada, levando em conta o período ideal em que a aprendizagem deve ocorrer e as maneiras pelas quais a aprendizagem pode ser alcançada da maneira mais eficaz, a partir de um custo e perspectiva centrados no aluno (Cook, 2007).

A versão online da $\mathrm{ABP}$ apresenta melhorias similares que correlacionam com diferentes aspectos de seu uso nos trabalhos selecionados, principalmente quando é possível ser observada a interação dos participantes, apesar de estarem separados no tempo e espaço. Pelo que foi contemplado na Tabela 2, é possível perceber que o uso desta ferramenta metodológica de ensino na EaD gerou benefícios manifestos no contexto de sua aplicabilidade, tais como: melhor gestão de tempo; desempenho do discente; aulas mais dinâmicas; aumento da participação e atenção dos alunos; análise mais críticas em solucionar e apresentar hipóteses para as atividades propostas; melhor compreensão do conteúdo e; maior flexibilidade no eixo colaborativo de ensino.

Percebe-se que, diante do foi apresentado sobre a ABP, os papeis dos alunos e professores sofrem modificações, onde o aluno assume um papel mais ativo no que está sendo transmitido e ensinado, verificando suas ações e apresentando propostas a partir de conhecimentos prévios. Nesse processo, o professor e/ou tutor limita-se ao papel de um mediador do conhecimento. A ABP proporciona um ensino diversificado, com possibilidades mais aprofundadas nas atividades práticas, e aplicáveis dentro de um contexto de aprendizagem baseada em evidências com conhecimento adquirido.

A análise dos estudos desta revisão também identificou certas situações que merecem atenção quanto aos desafios encontrados diante de sua aplicabilidade na EaD. Os autores dos trabalhos selecionados destacaram, em vários momentos: limitações de recursos; carência de domínio técnico, habilidades e manuseio de ferramentas tecnológicas por parte dos participantes; resistência e a falta de entendimento do uso desta metodologia como parte complementar do ensino e aprendizagem.

Para Almeida, Hubert e Bonamigo (2018, p. 18), “[...] formatos educacionais inovadores são acessíveis e gerenciáveis de todos os lugares do mundo, porém, há necessidade de uma cuidadosa seleção de ferramentas e planejamento, 
preparação e apoio no domínio técnico" - ou seja, a pretendida aplicação da ABP exige decisões institucionais e pedagógicas de acordo com a estrutura desenvolvida em cursos e/ou disciplinas que são ministradas no formato online.

Diante dos resultados obtidos nos trabalhos da presente revisão, entende-se que tentativas como essa são exercício formidável e imprescindível, vez que uma perspectiva reflexiva do uso dessa metodologia, compreendendo vantagens e seus desafios, fortalece o percurso em busca de uma melhor condição educacional.

É oportuno frisar que, através da metodologia e iniciativa apresentadas, o aluno assume papel ativo nas mais variadas fontes interativas, o que permite desenvolver o pensamento crítico e a criatividade na elaboração de decisões e resolução de problemas. A opção pela utilização de novas tecnologias no desenvolvimento da Aprendizagem Baseada em Problemas na Educação a Distância coaduna para uma produção significativa de conhecimento para todos os envolvidos na prática pedagógica.

\section{Conclusão}

As análises realizadas revelam que a tradicional forma de ensino, voltada especialmente para a centralidade no professor - o qual apenas transmite o conhecimento ao aluno e ele por sua vez, apenas assimila o que é apresentado, sem questionamentos e sem desenvolvimento de um pensamento crítico - não mais se encaixa nas exigências do mundo contemporâneo, principalmente nas novas e mais dinâmicas relações de ensino e aprendizagem.

Assim, este trabalho de revisão integrativa de literatura, sobre o método de Aprendizagem Baseada em Problemas (ABP), empreendeu em análise de seu uso em diferentes cenários da Educação a Distância $(\mathrm{EaD})$, chegando à conclusão de que o uso deste é proveitoso e tem resultados de positivos manifestos, conforme descrito no decorrer do artigo, mesmo em diversas modalidades de ensino e em diferentes áreas de conhecimento.

Ademais, não se almeja neste trabalho definir que a ABP é solução adequada para todos os problemas da educação e do atual modelo educacional do país, mas certamente os resultados encontrados constataram que ela contribui com o objetivo de assistir alunos e professores no processo de ensino-aprendizagem e de construção de conhecimentos de uma maneira inovadora e com metodologia didática, capaz de encontrar soluções para muitos dos problemas até então apresentados, ao enriquecer a dinâmica de ensino e elevar de forma decisiva a participação dos alunos na construção do seu próprio saber.

Entre os benefícios encontrados e citados é possível observar que existe aparente consenso de que a metodologia da ABP é capaz de proporcionar melhorias de desempenho em relação aos aspectos de autonomia na busca por conhecimentos, melhor assimilação dos conteúdos e melhoria da capacidade de entender e solucionar problemas reais, além da flexibilidade possibilitada pelo método, que se apresenta como atrativa, por possibilitar ao aluno o estudo do conteúdo proposto em qualquer lugar e/ou hora, à sua conveniência.

Entretanto, por mais relevantes que se apresentem os desafios e benefícios encontrados neste estudo, acredita-se que ainda é necessário aprimoramento e investigação mais complexos sobre a ABP em diversas áreas do conhecimento na Educação a Distância, de modo a possibilitar sua aferição quanto à efetividade de suas contribuições na dinâmica educacional em diferentes contextos, afastada das ciências da saúde e do cenário para o qual foi originalmente idealizada.

\section{Referências}

Ercole, F. F., Melo, L. S. D., \& Alcoforado, C. L. G. C. (2014). Revisão integrativa versus revisão sistemática. Revista Mineira de Enfermagem, 18(1), 9-12. de Almeida, V. O., Silva, H. T. H., \& Bonamigo, A. W. (2018). Aprendizagem Baseada em Problemas na Educação a Distância e as Influências para Educação em Saúde: Uma Revisão Integrativa. Revista Brasileira de Aprendizagem Aberta e a Distância, 17(1).

de Almeida, C. R. S., Camargo, M. N., \& Camargo, L. B. (2016). Educação popular e aprendizagem baseada em problemas na ead: uma aplicação no curso de ciências sociais EaD/UNIMONTES. Revista Multitexto, 4(2), 20-26. 
Research, Society and Development, v. 11, n. 2, e54011226275, 2022

(CC BY 4.0) | ISSN 2525-3409 | DOI: http://dx.doi.org/10.33448/rsd-v11i2.26275

de Souza, P. R., \& de Andrade, M. D. C. F. (2016). Modelos de rotação do ensino híbrido: estações de trabalho e sala de aula invertida. Revista E-Tech: Tecnologias para Competitividade Industrial-ISSN-1983-1838, 9(1), 03-16.

Borochovicius, E., \& Tortella, J. (2014). Aprendizagem Baseada em Problemas: um metodo de ensino-aprendizagem e suas práticas educativas. Ensaio: Avaliação e Políticas Públicas em Educação, 22(83), 263-294. Recuperado de https://revistas.cesgranrio.org.br/index.php/ensaio/article/view/287

Botelho, L. L. R., de Almeida Cunha, C. C., \& Macedo, M. (2011). O método da revisão integrativa nos estudos organizacionais. Gestão e sociedade, 5(11), 121-136.

Bozic, N., \& Williams, H. (2011). Online problem-based and enquiry-based learning in the training of educational psychologists. Educational Psychology in Practice, 27(4), 353-364.

Chigona, A. (2018). Digital fluency: necessary competence for teaching and learning in connected classrooms. The African Journal of Information Systems, 10(4), 7

Diehl, L. A., Gomes, C. P., Sary, G. D. V., Rocha, H. C. G. D., Camargo, L. M. A., \& Gordan, P. A. (2012). Educação à distância em nefrologia na Amazônia: processos e resultados. Revista Brasileira de Educação Médica, 36, 550-556.

Gentili, P. (1996). Neoliberalismo e educação: manual do usuário. Escola SA: quem ganha e quem perde no mercado educacional do neoliberalismo. Brasília: CNTE, 9-49.

Gil, A. C. (2009). Didática do ensino superior. Atlas.

Hargreaves, A. (2003). O Ensino na Sociedade do Conhecimento: a educação na era da insegurança. Porto: Porto Editora.

de Jong, N., Verstegen, D. M. L., Tan, F. E. S., \& O'connor, S. J. (2013). A comparison of classroom and online asynchronous problem-based learning for students undertaking statistics training as part of a Public Health Masters degree. Advances in Health Sciences Education, 18(2), $245-264$.

Meirieu, P. (1999). Aprender... sim, mas como?. Artmed.

Mezzari, A. (2011). O uso da Aprendizagem Baseada em Problemas (ABP) como reforço ao ensino presencial utilizando o ambiente de aprendizagem Moodle. Revista brasileira de educação médica, 35, 114-121.

MOORE, M. K., \& a Distância, G. E. (2007). uma visão integrada. São Paulo: Thomson Learning.

Morán, J. (2015). Mudando a educação com metodologias ativas. Coleção mídias contemporâneas. Convergências midiáticas, educação e cidadania: aproximações jovens, 2(1), 15-33.

Bacich, L., \& Moran, J. (2018). Metodologias ativas para uma educação inovadora: uma abordagem teórico-prática. Penso Editora.

Mugnol, M. (2009). A educação a distância no Brasil: conceitos e fundamentos. Revista Diálogo Educacional, 9(27), $335-349$.

OLIVEIRA, E. D. S. (2010). Ação docente na educação a distância: competências do professor invisível. Tecnologia educacional, 39 (190), 15-26.

Oliveira, M. D. D. R. D. (2013). Aprendizagem baseada em problemas/projetos em ambiente online na perspectiva de educadores e educandos da Ciência dos Alimentos.

Paiva, M. R. F., Parente, J. R. F., Brandão, I. R., \& Queiroz, A. H. B. (2016). Metodologias ativas de ensino-aprendizagem: revisão integrativa. SANARERevista de Políticas Públicas, 15(2).

Portela, N. M., Costa, J. M. B. D. S., \& Magalhães, G. S. D. G. (2020). A experiência com o uso do e-learning na aprendizagem baseada em problemas de um curso de medicina.

Ribeiro, L. R. D. C. (2005). A aprendizagem baseada em problemas (PBL): uma implementação na educação em engenharia na voz dos atores

Richardt, C. D. C. L. T. (2021). Aprendizagem baseada em problemas (ABP): proposta de aplicação prática para o ensino de português na educação a distância

Santos, C. P., Costa, C. M., Bezerra, I. S. Q., da Silva Assunção, L. R., Westphalen, F. H., \& Fernandes, Â. (2016). Estratégias criativas no processo ensinoaprendizagem da Radiologia Odontológica. Revista da ABENO, 16(4), 40-50.

Seufert, S., \& Meier, C. (2016). From eLearning to digital transformation: A framework and implications for L\&D. International Journal of Corporate Learning (iJAC), 9(2), 27-33.

Souza, M. T. D., Silva, M. D. D., \& Carvalho, R. D. (2010). Revisão integrativa: o que é e como fazer. Einstein (São Paulo), 8, $102-106$.

Takata, Y., Stein, G. H., Endo, K., Arai, A., Kohsaka, S., Kitano, Y., ... \& Terasawa, H. (2013). Content analysis of medical students' seminars: a unique method of analyzing clinical thinking. BMC medical education, 13(1), 1-6. 